\title{
Altered sensitivity to mechanical stimulation during prolonged subcutaneous administration of endothelin-I in rats
}

This article was published in the following Dove Press journal: Journal of Pain Research

20 May 2009

Number of times this article has been viewed

\author{
Guy Hans ${ }^{1,2,3}$ \\ Kristof Deseure ${ }^{2,3}$ \\ Marcel Vercauteren ${ }^{1,2,3}$ \\ 'Multidisciplinary Pain Center; \\ 2Department of Anesthesiology, \\ Antwerp University Hospital, \\ Edegem, Belgium; ${ }^{3}$ Laboratory \\ of Anesthesiology and Pain Research, \\ University of Antwerp, Wilrijk, Belgium
}

Correspondence: Guy Hans Antwerp University Hospital, Multidisciplinary Pain Center, Wilrijkstraat 10, 2650 Edegem, Belgium

Tel +3238214945

Fax +32 38214586

Email guy.hans@uza.be

\begin{abstract}
Cancer pain is often difficult to treat. Growing evidence indicates that chemical mediators secreted by the tumor itself play an important role in the development of cancer pain. One such mediator, endothelin-1 (ET-1) is secreted by different tumor types. Studies have indicated that ET-1 induces spontaneous and evoked nociception in rodents and in humans. The focus of all these studies has always been on a single administration of ET-1. Such an acute exposure to ET-1 however bears little resemblance to the clinical condition in which cancer patients are exposed continuously for many months to increased levels of ET-1. To improve the knowledge of the pathological role of ET-1 in cancer, we developed an animal model of prolonged exposure to ET-1. Rats were exposed to subcutaneous administration of ET-1 for seven consecutive days, with a total amount of $67.4 \mathrm{nmol}$. On days $+2,+3,+5,+7$, and +10 sensitivity to von Frey hairs and to pin-prick stimulation were evaluated. Prolonged administration of ET-1 induced signs of mechanical allodynia on several time points. Although the administered doses were very small, prolonged administration of ET-1 seems to lead to a state of mechanical allodynia.
\end{abstract}

Keywords: endothelin-1, rat, chronic administration, von Frey hair, pin-prick, mechanical allodynia

\section{Introduction}

Pain associated with metastatic cancer is often debilitating and difficult to treat. ${ }^{1}$ Among all cancer patients, the prevalence of metastatic cancer pain may exceed $75 \%{ }^{2}$ These patients often require large systemic doses of analgesics (eg, opioids) that do not always provide complete pain relief and that frequently produce undesirable side effects. There is growing preclinical evidence that peripheral sensitization of nociceptors is involved in the generation and maintenance of cancer pain..$^{3-5}$ Chemical mediators that are synthesized by either cancer cells or other cell types present in the tumor stroma, such as immune cells, may contribute to the lowering of nociceptor threshold of activation. ${ }^{6-8}$ One candidate mediator for lowering this threshold of activation is the potent vasoconstrictor peptide, endothelin-1 (ET-1), whose expression levels are high in several types of tumors. ${ }^{9-12}$

Most efforts to establish a pathological role of ET-1 have focused on its role in cardiovascular disease (for reviews see Sugden and Clerk, ${ }^{13}$ Shiffrin ${ }^{14}$ ). Recently, ET-1 has been recognized as a proalgesic mediator that is involved in the pathophysiology of many different pain syndromes which range from inflammatory states, complex regional pain syndromes (CRPS), sickle cell disease to cancer. ${ }^{9,15-17}$ Furthermore, its nociceptive effects are independent from its vasoconstrictive effects. ${ }^{18}$ Application of ET-1 has been shown to induce nociceptive behavior in rodents, and 
pain in humans. ${ }^{15,18-21}$ The results of clinical studies have shown the existence of a correlation between the severity of the pain and plasma levels of endothelins in patients with prostate cancer. $^{22}$

Despite the abundance of preclinical reports, the experimental conditions of these studies often bear little resemblance with clinical pathological states. These experiments usually use a single administration of ET-1, whereas in pathological conditions, such as cancer, patients have chronically (uninterrupted) elevated ET-1 levels in their plasma. The results of human studies have reported that mean plasma ET-1 levels in patients with different cancerous conditions are between 4-5 pg/mL. ${ }^{12,23-25}$ Although preclinical studies have applied very diverse concentrations of ET-1, these are probably consistently higher than the observed levels in human pathological conditions. Picomolar doses of injected ET-1 that yield very low local ET-1 concentrations are probably closer to the plasma levels of endogenous ET-1 that are found in human pathological conditions. It should be noted that tissue levels of ET-1 could well be extremely important for pain induction in cancer syndromes. ET-1 is indeed released from endothelial cells in a polarized fashion into the direction of surrounding vascular tissue and thus, plasma levels of ET-1 are likely to be only a fraction of the levels in surrounding tissues and should perhaps be considered as a merely "spill over" from the tissue compartment.

Until now, only one study has investigated the nociceptive effects of repeated or prolonged administration of ET-1. ${ }^{26}$ These investigators reported that repeated administration of low doses of ET-1 in rats led to the development of an acute desensitization period that persist for up to 24 hours. In the absence of confirming data, we decided to further investigate the effects of chronic delivery of exogenous ET-1 on spontaneous and evoked pain sensations in rats. The results of our preliminary experiments (unpublished data) revealed that prolonged perineural administration of ET-1 quickly (within 24 hours) results in the development of an intense fibrotic reaction around the subcutaneous tip of the cannula. This fibrotic reaction can rapidly result in complete blockage of the cannula, thereby reducing its performance and preventing further delivery of ET-1. Based on these preliminary findings, we opted to use very low doses of ET-1 to avoid any confounding inflammatory response. The final dose of ET-1 was chosen in accordance with the results of previous ET-1-based behavioral studies. ${ }^{18,20,27,28}$

The present project was undertaken to further characterize the exact role of ET-1 in nociception. To this end, we assessed the effect of prolonged administration of minute doses of ET-1 on the perception of mechanically evoked nociception.

\section{Material and methods Experimental animals}

Twenty male Sprague-Dawley rats (180-200 g at arrival, Charles River ${ }^{\circledR}$ ) were used in this protocol. They were housed in pairs in conventional plastic cages $(24 \times 40 \times 15 \mathrm{~cm})$ in a rodent colony room whose room temperature was $21 \pm 1{ }^{\circ} \mathrm{C}$ and a relative humidity of $45 \pm 5 \%$. Water and food were available ad libitum. Rats were kept under a reversed 12:12 hour dark/light cycle (lights on at 20:00 hours) and were acclimated for at least three weeks to the housing conditions before testing. The rats were habituated to the test procedure (see later for detailed description) three times before baseline values were obtained. The experimental protocol was approved by the Animal Ethical Committee of the University of Antwerp, and the experiments were performed in accordance with the Directives of European Community Council on the use and care of laboratory animals.

\section{Experimental groups and drug administration}

Rats were randomly assigned into two groups. Group 1 received ET-1 and group 2 received saline. Rats were implanted with an osmotic pump delivering either ET-1 (24 $\mu \mathrm{g} /$ day) or saline. The total amount of ET-1 administered to the rats over seven days was 67.4 nmol. ET-1 solution was prepared by dissolving the dry compound in saline. Preliminary testing has confirmed the stability of the ET-1 solution in these pumps. Additionally, samples of the ET-1 solution, after being contained in the osmotic pumps during seven consecutive days, were afterwards injected into the rat's paw to check any remaining biological activity. Indeed, such injections induced the previously described paw-flinching syndrome, evidencing a remaining biological (nociceptive) activity of this ET-1 solution. Pumps (Alzet 2ML1) were filled by injecting the solution into the pumps using a $1 \mathrm{ml}$ sterile syringe and a specialized blunt needle. These pumps have a nominal pumping rate of $10.0 \mu \mathrm{l} / \mathrm{hr}$ and a nominal duration of seven days. In order to obtain immediate pumping after implantation, the pumps were primed before implantation, according to the guidelines provided by the manufacturer. The pumps were prefilled in the usual manner and placed in sterile $0.9 \%$ saline at $37{ }^{\circ} \mathrm{C}$ overnight. During this priming period the catheter was draped outside the beaker to avoid any mixing of solutions. 
The next morning the pump was removed from the saline and implanted immediately.

The pumps were implanted into the rats under anesthesia. To this end, the rats were placed in a chamber containing $4 \%$ isoflurane. When the rats lost consciousness, they were removed from the chamber, and anesthesia was maintained by $2.5 \%$ isoflurane delivered by a face mask. The dorsal area was shaved and then disinfected with Hibitane ${ }^{\circledR}$ (chlorhexidine $0.5 \%$ dissolved in $70 \%$ alcohol). A skin incision of approximately $2 \mathrm{~cm}$ made at the distal end of the scapula and a small subcutaneous pocket was created using hemostatic forceps. Ampicillin $\left(0.1 \mathrm{ml}\right.$ of Pentrexyl ${ }^{\circledR}$, $\mathrm{Na}$ ampicillin $1 \mathrm{~g}$ ) was then injected into the pocket and the pump was inserted with its opening facing towards the back of the rat and away from the incision site (tip of the catheter pointing towards the lumbar region of the rat). Finally, the incision was closed with stainless steel staples (Disposable skin stapler with auto-release Appose 35 Regular; SherwoodDavis and Geck, St. Louis, MO, USA). After seven days the pumps were removed under general anesthesia.

In order to observe possible hemodynamic effects of the injected ET-1, systolic blood pressure was measured noninvasively in all animals daily by tail cuff plethysmography (Letica 5100; PanLab, Barcelona, Spain). Blood pressure monitoring was performed according to standard procedures, described previously. ${ }^{29}$ Additionally, body weight was measured daily. Other clinical signs of distress were also followed, such as the appearance of red tears.

\section{Behavioral testing}

Rats were individually transported from the colony room to the test room ( $15 \mathrm{sec}$ trip) in a covered plastic cage without bedding $(24(\mathrm{l}) \times 14(\mathrm{w}) \times 17(\mathrm{~h}) \mathrm{cm})$. von Frey and pinprick testing were conducted in a darkened room in which light was provided by a $60 \mathrm{~W}$ red light bulb suspended $1 \mathrm{~m}$ above the test area and with a $45 \mathrm{~dB}$ background noise that was sufficient to decrease the interference of sudden external auditory stimuli. Testing of evoked behavior was always performed at the same moment, more precisely during late morning. An extensive training phase for the rats was performed, in order to obtain highly reproducible results. All rats were as such acclimated to the testing conditions (ie, manipulation and transportation of the rats, stay in the darkened room and in the test box, performance of mechanical testing, procedure of blood pressure measurement) during 10 days before the start of the actual experiment. Baseline data were obtained one day before pump implantation. Further data were obtained on post-implantation days $+2,+3$,
$+5,+7$, and +10 . Data that were collected on the tenth day were at approximately three days after the end of pump infusion and the removal of the pump. Behavioral testing was performed by the same examiner, who was blinded with respect to administration of ET-1 or saline. The code was broken after data entry and analysis.

\section{Local cutaneous sensitivity}

Responsiveness to mechanical stimulation of a dorsal area, approximately $25 \mathrm{~mm}$ caudal to the opening of the subcutaneous pump, was measured using a series of three von Frey filaments (Stoelting Co, Wood Dale, IL, USA): $2.150 \mathrm{~g}, 7.370 \mathrm{~g}$, and $46.540 \mathrm{~g}$ and a pin-prick (21 gauge needle bent at a $45^{\circ}$ angle). The cages of the rats were placed next to each other on a table and the experimenter sat in front of them. The scoring system used was adapted from Vos and colleagues ${ }^{30,31}$ to evaluate the reaction of the rats to the stimulation, and we have used this modified method previously in different behavioral and pharmacological studies. ${ }^{32-35}$ The response of an animal is analyzed according to different response categories: 0: no response, 1: detection, 2: withdrawal reaction, 3: escape/attack. Lower scores indicate a weak responsiveness to stimulation while higher scores indicate a strong responsiveness. Under normal conditions (nonoperated rats and in absence of sensitivity modifying drugs) rats will display mechanical sensory thresholds ranging from 0 (no response at all to stimulation) to 1 (detective response) to the above mentioned mechanical stimulations in this particular skin region. These mechanical stimulations should therefore be considered as absolutely nonpainful in nonpathological conditions. In order not to sensitize the animals too much by performing too many manipulations and (mechanical) stimulations we decided not to include stimulation of distal dermatomes into the current study protocol.

\section{Statistical analysis}

All statistical analyses were performed using SPSS for Mac (version 13.0.0; SPSS Inc., Chicago, IL, USA). In order to analyze data from mechanical stimulation, preliminary Kruskal-Wallis testing was performed in order to detect any time- or treatment-dependent differences between the different experimental groups. Comparisons between responses to mechanical stimulation (von Frey filaments and pin-prick) between experimental groups on a single time point were performed using the Mann-Whitney test. Exact significance [2*(1-tailed sig.)] was hereby calculated. The parameters of systolic blood pressure in both experimental groups at the 
individual time points were compared using an unpaired $t$-test. The significance level for this study was set at $5 \%$.

\section{Results}

\section{Hemodynamic and distress monitoring}

No rats displayed significant alterations in systolic blood pressure (SBP) during the course of the administration of ET-1 or saline. At the starting point of the experiment, SBPs of both experimental groups were comparable (108 $\pm 5 \mathrm{mmHg}$ ). Mean SBPs over seven days (during ET-1 and placebo administration) were not significantly different in ET-1-treated rats compared with those in placebo-infused rats (114 \pm 3 vs $111 \pm 2 \mathrm{mmHg}$ ). ET-1 and placebo-treated animals displayed identical increases in body weight. No signs of distress were observed in any rats.

\section{Cutaneous sensitivity to mechanical stimulation}

Chronic administration of low doses ET-1 led to changes in sensitivity to different types of mechanical stimulation. Stimulation with the smallest von Frey hair $(2.150 \mathrm{~g})$ did not induce significant changes in response scores compared to saline-treated rats (Figure 1A). However, stimulation with $7.370 \mathrm{~g}$ von Frey filament (Figure 1B) led to significantly increased response scores on day $3(p<0.05)$. Stimulation with the largest of the three von Frey hairs (46.540 g; Figure $1 \mathrm{C}$ ) resulted in significantly higher response scores in ET-1-treated animals on days 3, 5, and 7 compared to the control animals stimulated with the identical hair $(p<0.05)$. Finally, behavioral responses to pin-prick stimulation (Figure 2) displayed a somewhat similar time course with significantly increased responsiveness in ET-1-treated rats on days 3 and $5(p<0.05)$. Saline-treated animals displayed some changes in responsiveness to mechanical stimulation following the implantation of the pumps, but these changes always failed to reach statistical significance when compared to pre-implantation values ( $p>0.05)$.

\section{Discussion}

To the best of our knowledge, the results of the present study constitute the first report on the effects on evoked nociception of a prolonged local administration of endothelin-1 (ET-1) in rats. ET-1-treated animals displayed significant changes in sensitivity to mechanical stimuli. Although the temporal changes in evoked behavior were somewhat different for the various intensities of mechanical stimulation, significant alterations occurred particularly from the
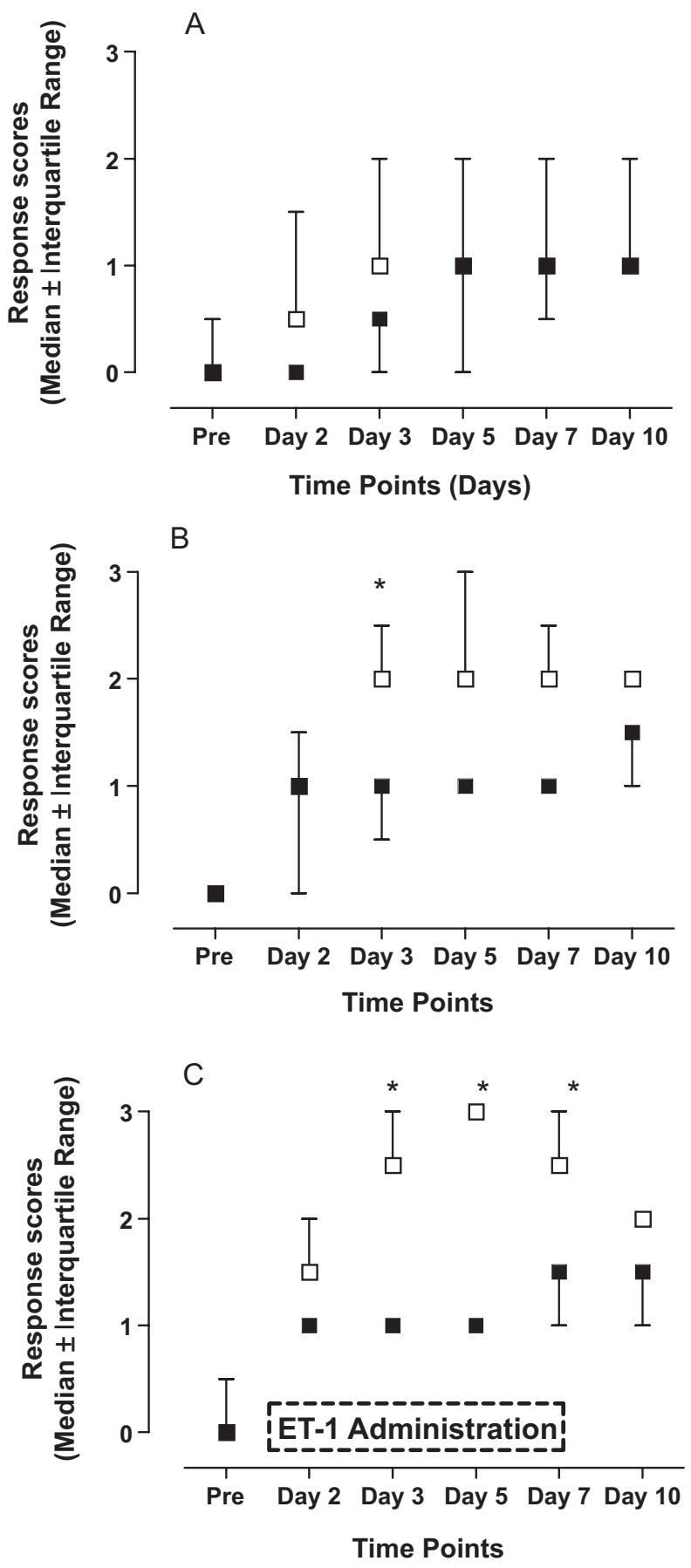

Figure I Time course of changes in response scores to (A) stimulation with 2.150 $\mathrm{g}$ von Frey filament, (B) $7.370 \mathrm{~g}$ von Frey filament, and (C) $46.540 \mathrm{~g}$ von Frey filament. Saline ( $\square)$ or endothelin-I (ET-I) ( $\square$ ) was administered for seven consecutive days (see box in graph C). The following response codes were applied: 'no response' (indicated by 0 in the X-axis of the graph), 'detection' (I), 'withdrawal reaction' (2), 'escape/attack' (3). Data are expressed as median values \pm interquartile range. Asterisks indicate a significant difference in response scores between ET-I administration and saline (Mann-Whitney $U$ test; $p<0.05$ ).

third day of ET-1 administration. The observed mechanical allodynia (increased sensitivity to nonpainful mechanical stimulations) disappeared after discontinuation of ET-1 administration. 


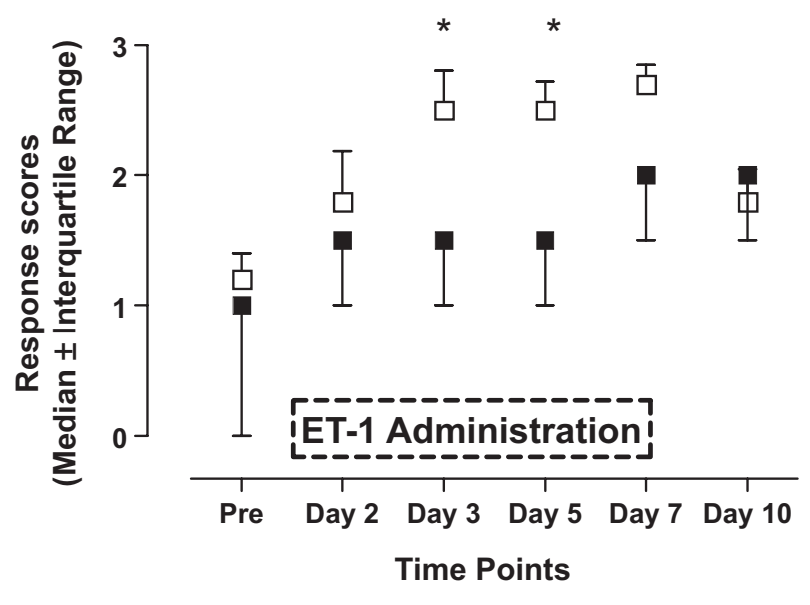

Figure 2 Effects of endothelin-I (ET-I) administration on the reaction to a pin-prick (2I gauge needle). Saline ( $\square$ ) or ET-I ( $\square$ ) was administered for seven consecutive days (see box). The following response scores were applied: 'no response' (indicated by 0 in the X-axis of the graph), 'detection' (I), 'withdrawal reaction' (2), 'escape/attack' (3). Data are expressed as median values \pm interquartile range. Asterisks indicate a significant difference between ET-I and saline administration (Mann-Whitney $U$ test; $p<0.05)$.

Rats receiving continuous administration of saline did display some changes in sensitivity to mechanical stimulation during the treatment period. However, none of these changes ever reached statistical significance and this result indicates that the administration of saline does not lead to significant changes in mechanical sensitivity. In addition, this result also means that the subcutaneous infusion of a fluid without pharmacological activity over seven days did not lead to the development of hypersensitivity in these animals. However, the subcutaneous implantation of a pump induces some changes in sensitivity in the animals, without ever reaching any significancy. Additionally, it should be noted that these changes occurred immediately after implantation of the pump, whereas the ET-1-induced changes occurred at a somewhat later stage. This time difference in the onset of changes in cutaneous sensitivity points to a clear difference between pump-induced and ET-1-induced changes.

The rather limited extent of changes in mechanical sensitivity that was observed in the ET-1-treated animals is probably due to the low doses of ET-1 used in this investigation. The administration of $24 \mu \mathrm{g}$ ET-1 per day corresponds to $9.6 \mathrm{nmol}$ per day (or a total of $67.4 \mathrm{nmol}$ over seven days). This means that only about 6 pmol of ET-1 was released subcutaneously each minute. Previous studies investigating the algesic effects of ET-1 have involved different methods of administering ET-1. These methods range from injecting 120 pmol ET-1 into the knee joint of rats, ${ }^{36}$ to application of $16 \mathrm{nmol} \mathrm{ET-1}$ onto the epineurial surface of the sciatic nerve of a rat. ${ }^{18}$ The results of a recent study have shown that single injection of $0.1 \mathrm{nmol}$ of ET-1 into the rat hind paw can induce sensitization of the ipsilateral paw to tactile stimulation by von Frey hairs. ${ }^{37}$ In our study, approximately $50 \%$ of this dose was delivered each minute by the subcutaneously implanted osmotic pump. Considering the results obtained from previously published dosing regimens of ET-1, it is quite remarkable that such a low dose of ET-1 used in our study indeed induces a prolonged state of mechanical hyperalgesia. This result implies that a continuous administration of ET-1 slowly leads to hypersensitivity, hereby providing an explanation for the onset of mechanical hyperalgesia after the third day of low dose ET-1 administration.

The experimental model used in our investigation reflects more accurately those clinical conditions in which patients are continuously exposed during weeks to months to elevated levels of ET-1. Nevertheless, some methodological limitations should certainly be considered. Animals were subjected to repeated mechanical stimulations that by itself could lead to some kind of hypersensitivity phenomenon. However, similar testing protocols have been used in our laboratory using other compounds and we found that the experimental protocols do not result in decreased thresholds. ${ }^{33,35}$ In addition, it would have been desirable to include additional testing for thermal sensitivity into the study protocol. However, this would have subjected the rats to even more testing manipulations on the same day, possible leading to a general state of sensitization and seriously jeopardizing the validity of the behavioral results. Because few data are currently available regarding the ET-1 concentrations that are present in cancerous tissues, the exact physiological relevance of the amounts of ET- 1 that were applied in this protocol can not be correctly evaluated. It would have been interesting to combine the parameters of evoked sensation with measurements of (plasma/tissue) ET-1-concentration. This was not done in this protocol due to the technical difficulties linked to measurements of ET-1 levels. Additionally, repetitive blood sampling would have greatly increased the stress for the animals. In future experiments we plan to use enzyme-linked immunosorbent assays to obtain ET-1 measurements in plasma and tissue. Finally, we opted not to use a cancer pain model, in order to exclude any possible confounding factors due to the presence of a growing tumor. Studies using different cancer pain models have already shown the development of reduced thresholds to mechanical stimulation, as well as pain reduction after administration of ET-1 antagonists. ${ }^{38-40}$ However, a growing tumor mass will result in many pathophysiological changes in the rodents, hereby possibly confounding the nociceptive effects of ET-1. 


\section{Conclusion}

This report is the first description of the neurosensory changes that develop following prolonged administration of ET-1. Although the administered doses were very small, prolonged administration of ET-1 seems to lead to a state of mechanical and heat hyperalgesia. This ET-1-based animal model could prove to be of great value for future investigations into the pathophysiology of ET-1-induced pain syndromes because the model, more closely mimics human clinicopathological conditions. Further studies are warranted to establish a doseresponse relationship of chronic delivery of ET-1 and to evaluate the effects of longer administration periods as well as different routes of administration. Finally, the differential effects of chronic administration of ET-1 on spontaneous behavior and evoked nociception should be carefully investigated in future experiments.

\section{Acknowledgments}

The authors gratefully acknowledge the financial support by the 'Benoît Foundation'. The authors report no competing or conflicts of interest in this work.

\section{References}

1. Mantyh PW. Cancer pain and its impact on diagnosis, survival and quality of life. Nat Rev Neurosci. 2006;7:797-809.

2. Portenoy RK, Payne D, Jacobsen P. Breakthrough pain: characteristics and impact in patients with cancer pain. Pain. 1999;81:129-134.

3. Honore P, Luger NM, Sabino MA, et al. Osteoprotegerin blocks bone cancer-induced skeletal destruction, skeletal pain and pain-related neurochemical reorganization of the spinal cord. Nat Med. 2000;6: 521-528.

4. Luger NM, Honore P, Sabino MA, et al. Osteoprotegerin diminishes advanced bone cancer pain. Cancer Res. 2001;61:4038-4047.

5. Schwei MJ, Honore P, Rogers SD, et al. Neurochemical and cellular reorganization of the spinal cord in a murine model of bone cancer pain. J Neurosci. 1999;19:10886-10897.

6. Peters CM, Lindsay TH, Pomonis JD, et al. Endothelin and the tumorigenic component of bone cancer pain. Neuroscience. 2004;126: 1043-1052.

7. Sabino MA, Mantyh PW. Pathophysiology of bone cancer pain. J Support Oncol. 2005;3:15-24.

8. Sabino MC, Ghilardi JR, Feia KJ, et al. The involvement of prostaglandins in tumorigenesis, tumor-induced osteolysis and bone cancer pain. J Musculoskelet Neuronal Interact. 2002;2:561-562.

9. Davar G. Endothelin-1 and metastatic cancer pain. Pain Med. 2001; 2:24-27.

10. Kurbel S, Kurbel B, Kovacic D, et al. Endothelin-secreting tumors and the idea of the pseudoectopic hormone secretion in tumors. Med Hypotheses. 1999;52:329-333.

11. Nelson JB, Carducci MA. The role of endothelin-1 and endothelin receptor antagonists in prostate cancer. BJU Int. 2000;85(Suppl 2): $45-48$.

12. Shankar A, Loizidou M, Aliev G, et al. Raised endothelin 1 levels in patients with colorectal liver metastases. Br J Surg. 1998;85: 502-506.

13. Sugden PH, Clerk A. Endothelin signalling in the cardiac myocyte and its pathophysiological relevance. Curr Vasc Pharmacol. 2005;3: 343-351.
14. Schiffrin EL. State-of-the-Art lecture. Role of endothelin-1 in hypertension. Hypertension. 1999;34:876-881.

15. Ferreira SH, Romitelli M, de Nucci G. Endothelin-1 participation in overt and inflammatory pain. J Cardiovasc Pharmacol. 1989;13(Suppl 5): S220-S222.

16. Groeneweg JG, Huygen FJ, Heijmans-Antonissen C, Niehof S, Zijlstra FJ. Increased endothelin-1 and diminished nitric oxide levels in blister fluids of patients with intermediate cold type complex regional pain syndrome type 1. BMC Musculoskelet Disord. 2006;7:91.

17. Hammerman SI, Kourembanas S, Conca TJ, Tucci M, Brauer M, Farber HW. Endothelin-1 production during the acute chest syndrome in sickle cell disease. Am J Respir Crit Care Med. 1997;156:280-285.

18. Davar G, Hans G, Fareed MU, Sinnott C, Strichartz G. Behavioral signs of acute pain produced by application of endothelin-1 to rat sciatic nerve. Neuroreport. 1998;9:2279-2283.

19. Chichorro JG, Zampronio AR, Rae GA. Endothelin ET(B) receptor antagonist reduces mechanical allodynia in rats with trigeminal neuropathic pain. Exp Biol Med (Maywood). 2006;231:1136-1140.

20. Gokin AP, Fareed MU, Pan HL, Hans G, Strichartz GR, Davar G. Local injection of endothelin-1 produces pain-like behavior and excitation of nociceptors in rats. J Neurosci. 2001;21:5358-5366.

21. Piovezan AP, D’Orleans-Juste P, Frighetto M, Souza GE, Henriques MG, Rae GA. Endothelins contribute towards nociception induced by antigen in ovalbumin-sensitised mice. Br J Pharmacol. 2004;141:755-763.

22. Nelson JB, Hedican SP, George DJ, et al. Identification of endothelin-1 in the pathophysiology of metastatic adenocarcinoma of the prostate. Nat Med. 1995;1:944-949.

23. Arun C, DeCatris M, Hemingway DM, London NJ, O'Byrne KJ. Endothelin-1 is a novel prognostic factor in non-small cell lung cancer. Int J Biol Markers. 2004;19:262-267.

24. Arun C, London NJ, Hemingway DM. Prognostic significance of elevated endothelin-1 levels in patients with colorectal cancer. Int J Biol Markers. 2004;19:32-37.

25. Mai HQ, Zeng ZY, Zhang CQ, et al. Elevated plasma big ET-1 is associated with distant failure in patients with advanced-stage nasopharyngeal carcinoma. Cancer. 2006;106:1548-1553.

26. Fareed MU, Hans GH, Atanda A, et al. Pharmacological characterization of acute pain behaviour produced by application of Endothelin-1 to rat sciatic nerve. J Pain. 2000;1:46-53.

27. Khodorova A, Fareed MU, Gokin A, Strichartz GR, Davar G. Local injection of a selective endothelin-B receptor agonist inhibits endothelin-1-induced pain-like behavior and excitation of nociceptors in a naloxone-sensitive manner. J Neurosci. 2002;22:7788-7796.

28. Khodorova A, Navarro B, Jouaville LS, et al. Endothelin-B receptor activation triggers an endogenous analgesic cascade at sites of peripheral injury. Nat Med. 2003;9:1055-1061.

29. Bunag RD. Validation in awake rats of a tail-cuff method for measuring systolic pressure. J Appl Physiol. 1973;34:279-282.

30. Vos BP, Strassman AM, Maciewicz RJ. Behavioral evidence of trigeminal neuropathic pain following chronic constriction injury to the rat's infraorbital nerve. J Neurosci. 1994;14:2708-2723.

31. Vos BP, Hans G, Adriaensen H. Behavioral assessment of facial pain in rats: face grooming patterns after painful and non-painful sensory disturbances in the territory of the rat's infraorbital nerve. Pain. 1998;76:173-178.

32. Deseure K, Breand S, Colpaert FC. Curative-like analgesia in a neuropathic pain model: parametric analysis of the dose and the duration of treatment with a high-efficacy 5-HT(1A) receptor agonist. Eur $J$ Pharmacol. 2007;568:134-141.

33. Deseure K, Koek W, Adriaensen H, Colpaert FC. Continuous administration of the 5-hydroxytryptamine1A agonist (3-Chloro-4fluoro-phenyl)-[4-fluoro-4-[[(5-methyl-pyridin-2-ylmethyl) -amino]methyl]piperidin-1-yl]-methadone (F 13640) attenuates allodynia-like behavior in a rat model of trigeminal neuropathic pain. J Pharmacol Exp Ther. 2003;306:505-514.

34. Deseure K, Koek W, Colpaert FC, Adriaensen H. The 5-HT(1A) receptor agonist $\mathrm{F} 13640$ attenuates mechanical allodynia in a rat model of trigeminal neuropathic pain. Eur J Pharmacol. 2002;456:51-57. 
35. Deseure KR, Adriaensen HF, Colpaert FC. Effects of the combined continuous administration of morphine and the high-efficacy 5-HT1A agonist, F 13640 in a rat model of trigeminal neuropathic pain. Eur $J$ Pain. 2004;8:547-554.

36. De-Melo JD, Tonussi CR, D'Orleans-Juste P, Rae GA. Articular nociception induced by endothelin-1, carrageenan and LPS in naive and previously inflamed knee-joints in the rat: inhibition by endothelin receptor antagonists. Pain. 1998;77:261-269.

37. Balonov K, Khodorova A, Strichartz GR. Tactile allodynia initiated by local subcutaneous endothelin-1 is prolonged by activation of TRPV-1 receptors. Exp Biol Med (Maywood). 2006;231:1165-1170.
38. Pickering V, Jay Gupta R, Quang P, Jordan RC, Schmidt BL. Effect of peripheral endothelin-1 concentration on carcinoma-induced pain in mice. Eur J Pain. 2008;12:293-300.

39. Schmidt BL, Pickering V, Liu S, et al. Peripheral endothelin A receptor antagonism attenuates carcinoma-induced pain. Eur J Pain. 2007;11: 406-414.

40. Yuyama H, Koakutsu A, Fujiyasu N, et al. Inhibitory effects of a selective endothelin-A receptor antagonist YM598 on endothelin-1-induced potentiation of nociception in formalin-induced and prostate cancerinduced pain models in mice. J Cardiovasc Pharmacol. 2004;44(Suppl 1): S479-S482.

\section{Publish your work in this journal}

The Journal of Pain Research is an international, peer-reviewed, open access, online journal that welcomes laboratory and clinical findings in the fields of pain research and the prevention and managemen of pain. Original research, reviews, symposium reports, hypothesis formation and commentaries are all considered for publication.

\section{Dovepress}

The manuscript management system is completely online and includes a very quick and fair peer-review system, which is all easy to use. Visit http://www.dovepress.com/testimonials.php to read real quotes from published authors.

\footnotetext{
Submit your manuscript here: http://www.dovepress.com/journal-of-pain-research-journal
} 